\title{
Utilización de la Transferrina Deficiente en Carbohidratos (CDT) en el diagnóstico y seguimiento de la dependencia alcohólica
}

\author{
Pascual, F ${ }^{1}$; Cortés, M.T. ${ }^{2}$; Sastre, J. ${ }^{3}$. \\ Fundación A.E.P.A.. Unidad de Alcohología. Área de Salud de Alcoi. Conselleria de Sanitat. Generalitat Valenciana. \\ ${ }_{2}^{2}$ Profesora Facultad de Psicología Universidad de Valencia. \\ ${ }^{3}$ Servicio análisis Clínicos Hospital Virgen de los Lirios de Alcoi. Conselleria de Sanitat. Generalitat Valenciana.
}

Enviar correspondencia a:

Francisco Pascual Pastor. Unidad de Alcohología. El Camí nº 40 - 03801 - Alcoi (Alicante) Tfn. 965543047 FAX. 965543167 . E-Mail: fr.pascualp@coma.es

\section{RESUMEN}

Al no contar con una prueba de laboratorio que tenga una fiabilidad absoluta en el diagnóstico del alcoholismo y en la evolución posterior para corroborar el consumo o la abstinencia, se ha recurrido últimamente a la utilización del transferrina deficiente en carbohidratos para acercarnos al diagnóstico y a la evolución en el proceso terapéutico.

En la Unidad de Alcohología del Área de Salud de Alcoi de la Conselleria de Sanitat de la Generalitat Valenciana, venimos utilizando esta prueba de laboratorio desde el año 1997 hasta la actualidad. Creemos que una vez realizada esta prueba en más de 400 pacientes ha llegado el momento de realizar un análisis descriptivo y estadístico para poder valorar la utilidad de la misma, así como su fiabilidad, especificidad y sensibilidad.

Por medio del presente estudio hemos podido verificar la utilidad de la transferrina deficiente en carbohidratos tanto en el diagnóstico como en el seguimiento de los dependientes al alcohol. Lo que presenta a la CDT como uno de los mejores marcadores biológicos para el alcoholismo.

Palabras Clave. Alcoholismo, diagnóstico, evolución, CDT, GGT, VCM.

\section{SUMMARY}

As we don't dispose with a laboratory sample which have a total confidence in the diagnostic of the alcoholism and in the later evolution, in order to corroborate the consumption or the abstinence, we have had to apply, in the last instance, to the use of the deficient in carbohydrates transferrine in order to approach to the diagnosis and evolution in the therapeutic process.

We are using this experiment from 1997 to the present time at Alcoholism Department of the Health Area of Alcoi in the Council Health Office of the Valencian Community (Spain) .

We believe that once realised this experiment in more than 400 patients, has arrived the moment to carry out a descriptive and statistical analysis to be able to value the utility of itself, so that it confidence, specificity and sensibility.

This present study have let to check the very useful the deficient in carbohydrates transferrin in the alcoholism diagnosis and the follow - up.

The DCT is the best biological screening for the alcoholism.

Key words: Alcoholism, diagnostic, evolution, CDT, GGT, VCM.

\section{INTRODUCCIÓN}

$\mathbf{P}$ ara el diagnóstico y la evolución del trastorno por dependencia al alcohol hemos estado utilizando a nivel clínico la gamma glutamil transpeptidasa o transferasa (GGT) junto con la GOT y la GPT, además del Volumen Corpuscular medio de los hematíes.

No obstante el aumento de la GOT y la GPT nos están indicando un daño hepático que no tiene en principio porque corresponderse con una etiología etílica del mismo.

El incremento de la GPT o transaminasa glutámico pirúvica denominada últimamente ALT o Alanina aminotransferasa está indicando mayoritariamente disfunción hepática provocada por distintos cuadros como la hepatitis, cirrosis, necrosis hepática colestasis, isquemia hepática, tumor hepático y en el consumo de fármacos hepatotóxicos.(1)

En la elevación de la GOT o transaminasa glutámico oxalacética, también denominada AST o aspartato aminotransferasa, la dispersión clínica es todavía mayor. Esta elevada en el infarto agudo de miocardio, operaciones cardíacas, cateterismo y angioplastia del corazón, hepatitis, cirrosis hepática, pancreatitis 
aguda, traumatismo muscular esquelético cirugía reciente no cardíaca, traumatismos múltiples, necrosis hepática, quemaduras profundas graves, anemia hemolítica, distrofia muscular progresiva, mononucleosis infecciosa con hepatitis, convulsiones recientes, procesos infiltrativos hepáticos (tumores), enfermedades musculares primarias (miopatía, miositis) y enfermedad renal aguda.(1)

Algo similar ocurre con el aumento de la GGT, aunque se puede considerar que este último es un marcador más específico pues puede elevarse sin que haya perjuicio del parénquima hepático, como sucede en la esteatosis hepática muy frecuente entre los consumidores habituales y abusivos de bebidas alcohólicas. Pero tampoco una elevación de la GGT es exclusivamente determinante del alcoholismo, ya que este aumento puede ser debido a otras enfermedades como hepatitis, cirrosis, necrosis hepática, isquemia hepática, infarto agudo de miocardio (4 -10 días después), insuficiencia cardíaca congestiva, tumor hepático, fármacos hepatotóxicos, colestasis, ictericia y pancreatitis, no obstante y aunque se calcula que está incrementado en el 75\% de los alcohólicos los datos anteriormente expuestos le confieren una escasa especificidad y un escaso valor en el diagnóstico diferencial.(1)

La GGT es una enzima glicoproteica que se encuentra en las membranas celulares de numerosos tejidos, particularmente en los especializados en los mecanismos de absorción o secreción. En el hígado se encuentra en las membranas del hepatocito y en los microsomas de las células, especialmente en las situadas en los conductillos biliares. La actividad sérica de esta enzima, al menos en un sujeto normal, es de origen hepático.(2)

En cuanto al aumento del volumen corpuscular medio (VCM) lo único que refleja es un aporte suficiente de $\mathrm{O} 2$ a la sangre y por lo tanto la hemoglobina debe hacer acopio del mismo elevando el tamaño de los glóbulos rojos, hecho que es frecuente en cuadros de distrés respiratorio o de tabaquismo.

Se suele utilizar para clasificar las anemias y está también incrementado en las enfermedades hepáticas, tratamiento con antimetabolitos, anemia perniciosa por descenso de vit. B12 y en la disminución del ácido fólico, muy frecuente en el alcoholismo además de otras enfermedades.(1)

Con todos estos datos podemos afirmar que no disponemos de una prueba de laboratorio patognomónica para el diagnóstico del alcoholismo, aunque las dos pruebas de laboratorio más utilizadas hayan sido el VCM y la GGT, siendo considerada esta última la de mayor utilidad actualmente.

Desde hace aproximadamente una década se está utilizando la Transferrina deficiente en carbohidratos para aproximarnos de un modo más preciso al diag- nóstico clínico de la dependencia al alcohol. A pesar del tiempo transcurrido diversos estudios no se ponen de acuerdo a la hora de precisar la utilidad práctica de su determinación.

La ingesta crónica de alcohol, se ha comprobado que induce isoformas de CDT, disminuyendo la carga eléctrica negativa por disminución de residuos terminales de ácido siálico, elevándose como consecuencia el punto isoeléctrico, lo que es aprovechable para su detección por técnicas de separación tales como la cromatografía en columna y posteriormente cuantificación por enzimomunoensayo, técnica ésta utilizada en el laboratorio para su determinación.

La CDT sérica aparece anormalmente elevada en la mayoría de los pacientes que consumen más de 50 gramos de etanol al día durante una semana.

Los valores se normalizan a las dos semanas de abstinencia, volviéndose a elevar ante consumos que cumplan la condición de al menos 50 gr./ día durante una semana.

Durante el embarazo existe una relación positiva entre la semana de gestación y la concentración de CDT, por lo que deberá tenerse en cuenta este extremo al interpretar el valor de este parámetro.

Parece ser que estaría elevada en el $80 \%$ de bebedores de riesgo, y hasta el 90\% en alcohólicos. Siendo útil en la monitorización de abstinencia y detección de recaídas. Pero su especificidad se situaría entre el 72-99\% y su sensibilidad se tendría un rango del 31 al $81 \%$ (Rubio; G. 1998) (3), además apunta que para que aumente el CDT los consumos de etanol se establecen entre 60- 80 gr/día al menos durante 1 semana.

Según Saiz, P.A y cols. (4) la Especificidad sería de un $70-100 \%$ y la Sensibilidad de $69-100 \%$. Estos autores explican además, que tanto la especificidad como la sensibilidad Ilegan al 100\% cuando coexiste un incremento de la GGT y de la CDT en consumidores de más de 60 gr. / día. Las cifras de CDT se normalizaría a las dos semanas de la abstinencia.

En el XIX Congreso ISBRAS (Society for Biomedical Researsch on Alcoholism) celebrado en Copenhague en Julio de 1998 se presentaron diversos artículos al respecto dando distintas cifras de especificidad $82 \%$ y sensibilidad 97\% (Salesporo, M. 1998) (5) considerándolo como un buen marcador de recaídas.

En pacientes cirróticos el CDT se mantiene elevado a pesar de la abstinencia y tarda mucho en disminuir (Gordon, H.M. 1998). (6)

También se ha demostrado que no existe correlación entre la transferrina total y la CDT (J. Van Pelt. 1998) (7). Ni tampoco se ha encontrado una alteración de los receptores lipoprotéicos de membrana ante un aumento de la transferrina deficiente en carbohidratos. (Raja K.O. et alti. 1998).(8) 
No obstante no existe una unanimidad a la hora de valorar la utilidad de la CDT, así en un estudio realizado en 44 mujeres gestantes consumidoras de bebidas alcohólicas y con un grupo control de 68 embarazadas se concluyó que la GGT y el VCM eran más eficaces como marcadores de laboratorio para la detección del consumo abusivo de alcohol y sobre los efectos del alcohol en el feto (9).

En una revisión de 54 estudios (10) sobre la CDT en comparación con otros marcadores de consumo de alcohol, 2 de ellos consideraban la CDT más sensible que la GGT si el consumo se había realizado entre las 3 ó 4 últimas semanas, 6 estudios avalaban su uso como marcador de recaídas en el consumo, 7 estudios demostraron más sensibilidad en general que la CDT, 5 estudios encontraron diferencias a favor de la CDT pero sin significación estadística, en cambio 3 estudios dudaban de la especificidad y sensibilidad de la prueba, otros 7 arrojaban una mayor sensibilidad para la GGT.

De todos los estudios solo 3 incluyeron mujeres y en 8 estudios se llega a admitir que la aumenta tanto CDT en hombres y en mujeres con enfermedad hepática.

Existen también 7 estudios realizados en atención primaria pero solo entre grandes bebedores en los que si que se demuestra su utilidad.

Otro estudio (11) realizado en 1999 da unas cifras de sensibilidad del $92 \%$ y de especificidad del $80 \%$, en cambio en este caso se dice que el incremento de la CDT es mayor en mujeres que en varones, no se influencia por el uso de disulfiram y puede ser utilizado en pacientes con cirrosis.

Un trabajo publicado en mayo del 2000 por BrÀthen (12) en que analiza a 158 sujetos alcohólicos (83 hombres y 75 mujeres) todos ellos con el AUDIT positivo, concluye con una sensibilidad del $41 \%$ y una especificidad del $84 \%$, encontrando mayor sensibilidad para las mujeres.

Según Mundle (sep.1999) (13) la GGT tiene más sensibilidad que la CDT, con una p menor de 0'001, pero la utilización concomitante de la GGT y la CDT alcanza una sensibilidad del $90 \%$, cuando la GGT solo tiene niveles del 33\% después de cuatro días de abstinencia y del $72 \%$ para 4 días antes.

Otros dos trabajos (14) aprecian un aumento en la sensibilidad para ingestas superiores a $60 \mathrm{gr}$. de etanol al día y para períodos de abstinencia cortos incrementándose la utilidad en Bebedores excesivos regulares $(B E R)$ y siendo de escaso rendimiento diagnóstico en bebedores intermitentes (BIR) (15).

En resumen, entre los pacientes alcohólicos la CDT presenta varias ventajas, es muy específica y tiene pocos falsos positivos, pero también tiene algún inconveniente como son a priori una menor sensibilidad en varones de edad avanzada, el consumo espo- rádico y el consumo diario a dosis bajas, donde se hace difícil descubrir recaídas en sujetos dependientes en abstinencia (2). Todo ello nos ha llevado a realizar el presente estudio.

En nuestro estudio la CDT se determinó por medio de un enzimomunoensayo competitivo, habiéndose separado previamente las isoformas de transferrina presente en la muestra mediante cromatografía de intercambio en microcolumnas, aprovechando la carga eléctrica y puntos isoeléctricos más elevados de lo normal, quedando ésta en eluido, mientras que las demás isoformas de la transferrina son retenidas en columna. (2-16)

La CDT del eluido compite con una cantidad fija de transferrina marcada enzimáticamente por los sitios de unión de anticuerpos monoclonales antitransferrina. Estos anticuerpos se unirán a los anticuerpos antirratón de oveja que revisten la superficie de los micropocillos de la microplaca.

Los resultados se expresan en unidades arbitrarias. (2)

\section{MATERIALY MÉTODOS}

\section{Descripción de la muestra}

La muestra total está compuesta por un total de 432 participantes de los cuales el $82.4 \%$ son varones y el $17.6 \%$ son mujeres con edades comprendidas entre 19 y 76 años. En este primer momento, un $33,3 \%$ de los participantes están abstinentes $(n=144)$, frente al 66,7\% ( $n=288)$ que continúan consumiendo.

La abstinencia para todos los casos se ha corroborado con controles periódicos con el alcoholímetro y con la información contrastada con los familiares.

Los valores normales de la GGT en nuestro laboratorio se sitúan en los límites inferiores a $49 \mathrm{U} / \mathrm{L}$ para los hombres y de $32 \mathrm{U} / \mathrm{L}$ para las mujeres.

En cuanto al Volumen Corpuscular Medio el valor máximo para ambos sexos es de $97 \mathrm{fL}$.

Y para la CDT los valores normales son inferiores a $14 \mathrm{U} / \mathrm{L}$ en las pruebas realizadas antes del año 2000 y en valores inferiores a $6 \mathrm{U} / \mathrm{L}$ en los determinados después del 1 de enero del 2000. El coste de la determinación es de 1.300 pts.

Los valores hallados en la primera determinación quedan reflejados en la tabla I.

En nuestro estudio se incluyeron pacientes de ambos sexos, tanto en la primera medida como a los 6 meses.

Transcurridos 6 meses del inicio del tratamiento, las analíticas fueron realizadas a 91 de los participantes, $78 \%$ varones y $22 \%$ mujeres, un $47 \%$ estaban 
abstinentes (43), frente a un 53\% (48) que seguían consumiendo.

Esta segunda determinación realizada a los 6 meses está constituida por 91 pacientes que seguían en tratamiento y se habían realizado los análisis correspondientes para poder entrar en el estudio, del resto el 30\% había abandonado tratamiento y de los otros no contábamos con determinaciones analíticas que nos permitiesen incluirlos en el estudio.

Las Medias y desviaciones típicas de los tres índices a los seis meses de tratamiento las podemos observar en la tabla II

Tabla I: Medias y desviaciones típicas de los tres índices en la primera muestra

\begin{tabular}{|l|c|c|c|c|c|}
\hline & $\mathbf{N}$ & Media & Desv. Típ. & Mínimo & Máximo \\
\hline GGT & 356 & Hombres: 78,919 & 156,320 & 9 & 1.655 \\
(U/L) & 76 & Mujeres: 74,000 & 141,731 & 7 & 1.091 \\
\hline VCM & 432 & 94,019 & 5,427 & 64,6 & 110 \\
(fL) & & & & & \\
\hline CDT & 363 & Antes 2000: 23,105 & 16,567 & 1,9 & 104,7 \\
(U/L) & 69 & Después: 5,033 & 2,668 & 1,4 & 19,7 \\
\hline
\end{tabular}

Tabla II: Medias y desviaciones típicas de los tres índices a los seis meses de tratamiento.

\begin{tabular}{|c|c|c|c|c|c|}
\hline & $\mathbf{N}$ & Media & Desv. Típ. & Mínimo & Máximo \\
\hline GGT & 71 & Hombres: 60,676 & 97,577 & 9 & 704 \\
(U/L) & 20 & Mujeres: 33,400 & 24,552 & 10 & 116 \\
\hline VCM & 91 & 93,113 & 4,965 & 76,2 & 103 \\
(fL) & & & & & \\
\hline CDT & 71 & Antes 2000: 22,669 & 16,829 & 2,5 & 110 \\
(U/L) & 20 & Después: 5,125 & 2,012 & 2,8 & 9 \\
\hline
\end{tabular}

En el grupo analizado a los 6 meses se observa un mayor porcentaje de personas que han conseguido la abstinencia pero sobre todo podemos ver como los valores medios de laboratorio para cada una de las tres variables han disminuido considerablemente, lo que nos indicaría que incluso entre los bebedores ha habido una disminución en las cantidades ingeridas.

\section{Procedimiento}

La recogida de la información se inició en 1997, incluyendo evaluaciones de los primeros seis meses de tratamiento en sujetos que acudían a la UA de Alcoy. Algunos de los sujetos analizados tienen hasta un total de 8 análisis realizados, pero hemos querido sólo considerar las medidas tomadas en la primera ocasión y transcurridos 6 meses.
El porcentaje de abstinentes encontrados en la primera prueba (primer análisis) es debido a que se tomaron también para el estudio pacientes que estuviesen ya en tratamiento en la Unidad. El control a los 6 meses suele coincidir con el tercer control que realizamos en nuestra Unidad sobre todo cuando se han observado alteraciones iniciales.

La recogida de datos se ha prolongado hasta finales del año 2000. Concretamente, la muestra inicial está compuesta por 432 sujetos que han iniciado su tratamiento en momentos temporales distintos, pero a los que se ha evaluado a lo largo de sus primeros seis meses de intervención, realizando para el estudio dos medidas: una al inicio de ésta y la otra a los seis meses, esta última se ha realizado a 91 de los 432 iniciales, ya que algunos abandonaron tratamiento y el resto (la mayoría) tenían una evolución que no requería nuevos controles analíticos al no presentar alteraciones importantes.

La primera medida se suele realizar al ingreso, la segunda a los dos meses y la tercera a los 6 meses de tratamiento.

A los participantes se les ha realizado una analítica en la que se han evaluado distintos índices de consumo de alcohol (VCM, GGT y CDT) con objeto de determinar cual de ellos es el más discriminativo.

\section{Pruebas estadísticas utilizadas. (17)}

Nos hemos servido de diferentes pruebas, desde el cálculo de frecuencias y descriptivos más generales (media, desviación típica, puntuación mínima y máxima...) para conocer las características de la población con la que se trabaja, hasta el análisis de varianza para determinar posibles diferencias en los índices cuantitativos CDT, VCM y GGT (VDs) en función de la edad, el sexo, el tipo de bebedor y el estar o no abstinente (VIs). Concretamente el análisis de varianza nos permite determinar en qué medida las diferencias entre las medias de las variables están relacionadas con la pertenencia de los sujetos a grupos distintos.

* Dependiendo de la homogeneidad de la muestra (cuando la prueba de Levene ha resultado significativa), recurrimos en algunas ocasiones a ANOVAs más simples y en su defecto a pruebas $\mathbf{t}$ (diferencias entre las medias de dos grupos) o incluso a pruebas no paramétricas ( $U$ de Mann-Whitney).

Otro de los objetivos perseguidos en este trabajo era determinar cual de los tres índices era el más discriminativo de la población o incluso qué combinación de estos permitiría clasificar con mayor precisión a los participantes. Para ello nos servimos del análisis discriminante.

Si sólo utilizamos una variable y dos grupos, basta con examinar las medias y varianzas de los grupos 
directamente y describir las diferencias entre ellos. Pero, cuando lo que se pretende es utilizar dos o más variables combinadas de tal modo que una de esas posibles combinaciones muestre el máximo de diferencias entre las medias de las puntuaciones de los grupos y el menor solapamiento, entonces hemos de aplicar el análisis de función discriminante.

El objetivo es hallar la mejor combinación de variables (los índices CDT,VCM,GGT) que nos permitan discriminar entre consumidores y no consumidores. Será esta combinación (también puede ocurrir que el resultado nos indique que lo mejor es una variable simple) la que nos permitirá predecir con un mínimo de error un criterio (análisis de canónico) y clasificar a los sujetos en grupos mutuamente excluyentes.

En definitiva nos planteamos si los índices CDT, VCM y GGT permiten clasificar y predecir de forma clara, en virtud de las puntuaciones obtenidas en dichas variables, a consumidores y abstinentes en grupos excluyentes y exhaustivos.

\section{RESULTADOS}

\section{Primera determinación:}

- VCM/ Abstinencia *edad (significativo únicamente el factor principal abstinencia)

Respecto al índice VCM únicamente se aprecian diferencias significativas entre los sujetos en función de la variable ABSTINENCIA. Esto permite afirmar que el VCM únicamente varía significativamente en función de si los sujetos están o no consumiendo.

Abstinente: Frecuencia 144 (valor medio 92'57 fL), Consumidor: Frecuencia 288 (valor medio 94'74 fL)

El análisis de varianza realizado con la variable abstinencia, nos indica que existen diferencias significativas entre consumidores y abstinentes en el índice VCM ( $F 1,430=15,73 ; p=0,005)$. El índice VCM es superior entre los consumidores.

VCM no discrimina en función del sexo (hombres y mujeres obtienen puntuaciones similares en este índice), ni tampoco en función de la edad (obtienen puntuaciones similares los sujetos mayores y menores a 40 años).

La variable GGT la hemos diferenciado en función del sexo, pues este marcador así lo exige.

Los resultados a los que se llega es que en los varones esta variable no permite diferenciar en función de la edad ni tampoco de estar o no abstinente.

Las puntuaciones en GGT que obtienen los varones que constituyen esta primera muestra son similares independientemente de la edad que tengan y de estar o no consumiendo.
El mismo resultado se mantiene para las mujeres. En este caso tampoco las puntuaciones en GGT varían ni en función de la edad ni tampoco de estar o no abstinentes.

Si tomamos el índice GGT sin diferenciar entre hombres y mujeres, sí que se aprecian diferencias entre los consumidores y los abstinentes.

Esto nos está cuestionando la diferencia que se establece de este índice en función del sexo. Parece ser que la variable clave no es el sexo sino la abstinencia.

\section{- GGT/ Abstinencia (sin diferenciar en función} del sexo)

Valor GGT en función de la abstinencia. Abstinente: Frecuencia 144 (valor medio 52'64 U/L). Consumidor: Frecuencia 288 (valor medio 90' $75 \mathrm{U} / \mathrm{L}$ )

El análisis de varianza, prueba t y U de ManWhitney (sería el más adecuado mencionar por cuestiones de homogeneidad) realizado con esta variable, nos indica que existen diferencias significativas entre consumidores y abstinentes en el índice GGT. El índice GGT es superior entre los consumidores.

Respecto a la variable CDT en el período previo al año 2000, el cual engloba las primeras evaluaciones realizadas en 1997, 1998 y 1999, observamos variaciones en función de permanecer o no abstinente.

El mismo resultado se desprende del análisis realizado con los primeros casos evaluados a lo largo del año 2000:

- CDT/edad*abs*sexo (en ambos casos: no homogénea pero abstinencia es significativa)

- CDT/abs*edad (en ambos casos: no homogénea pero abstinencia es significativa)

- CDT/abs*sexo (en ambos casos: no homogénea pero abstinencia es significativa)

- CDT/abs (U Mann-Whitney): significativa la abstinencia en ambos casos. Antes de 2000; U de Mann-Whitney 2023,000; $p=0$ 000

AÑO 2000 U de Mann-Whitney 20,500; p= 0'000

Existen por tanto diferencias significativas en las puntuaciones obtenidas en el índice CDT en función de estar o no abstinente. Los consumidores muestran niveles mayores de CDT. Los puntos de corte están en 14 U/L hasta el 2000 y en 6 después del 2000, valores superiores serían indicativos de consumo.

\section{- CDT (antes 2000) /Abstinencia}

Abstinentes: Frecuencia 85 (valor medio 10'709 U/L). Consumidores: Frecuencia 278 (valor medio 26'895 U/L). 


\section{- CDT (después 2000) /Abstinencia}

Abstinentes: Frecuencia 59 (valor medio 4'356 U/L). Consumidores: Frecuencia 10 (valor medio 9'030 $U / L)$.

Podemos concluir que en este primer momento es la variable abstinencia la que parece tener una mayor repercusión frente al sexo, año de realización de la prueba y edad del participante a la hora de hacer variar los resultados obtenidos en los marcadores de consumo de alcohol, principalmente VCM y CDT.

\section{Determinación a los 6 meses:}

A los seis meses de tratamiento se vuelven a repetir las analíticas obteniéndose diferencias en cuanto a la adecuación de las pruebas respecto al primer análisis realizado.

\section{- VCM/sexo*edad*abs}

\section{- VCM/abs*edad*bebedor}

En ambos casos es significativa la variable abstinencia ( $F 1,81=4,722 ; p=0,033 ; F 1,81=6,233 ; p=0,015)$. En este momento el estar o no abstinente continua discriminando entre los sujetos con mayores o menores niveles de VCM, siendo éste mayor entre los consumidores.

Valor VCM en función de la abstinencia. Abstinente: frecuencia 43 (valor medio 91'56 fL). Consumidores: 48 (valor medio 94'51 fL).

Los consumidores obtienen medias significativamente más altas en VCM que los abstinentes.

- VCM/sexo*edad*bebedor (es significativa la interacción sexo*bebedor)

Encontramos más diferencias entre los sujetos en este índice en función de la interacción sexo*bebedor $(F 1,81=6,115 ; p=0,015)$. Parece ser que las mujeres con un consumo intermitente puntúan significativamente más alto en esta variable que las mujeres bebedoras excesivo regulares (ver gráficos).

Contrariamente entre los varones no aparecen diferencias significativas a nivel estadístico, aunque puede apreciarse una relación inversa respecto a las mujeres.

En este caso, los varones BER obtienen puntuaciones mayores en VCM que los varones bebedores intermitentes (BIR). ( Gráfico I)

VCM/sexo*abs*bebedor (es significativa la abstinencia $(F 1,81=4,349 ; p=0,040)$; la interacción sexo *bebedor $(F 1,81=4,028 ; p=0,048)$. y la interacción triple).

En este caso ha resultado significativa la interacción sexo*abs*bebedor $(F 1,81=4,624 ; p=0,034)$. lo que nos permite extraer nuevas conclusiones. (Gráfico II y $|I|))$

\section{Medias marginales estimadas de VCM}

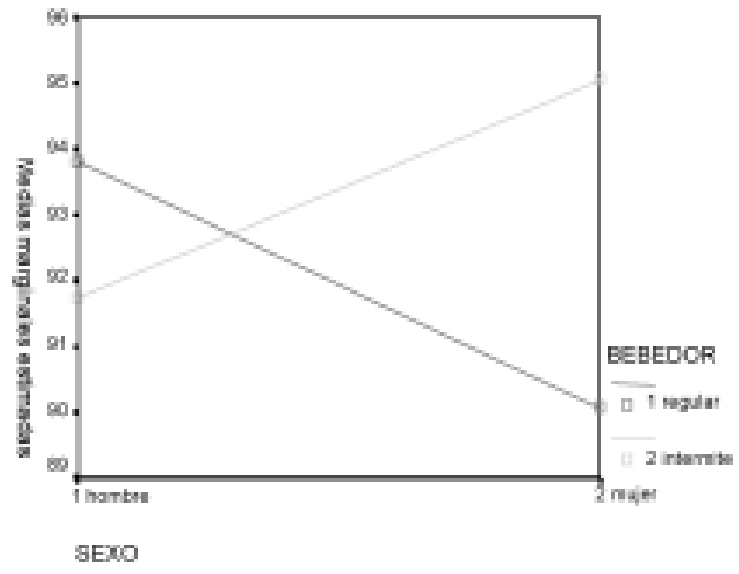

Gráfico I.

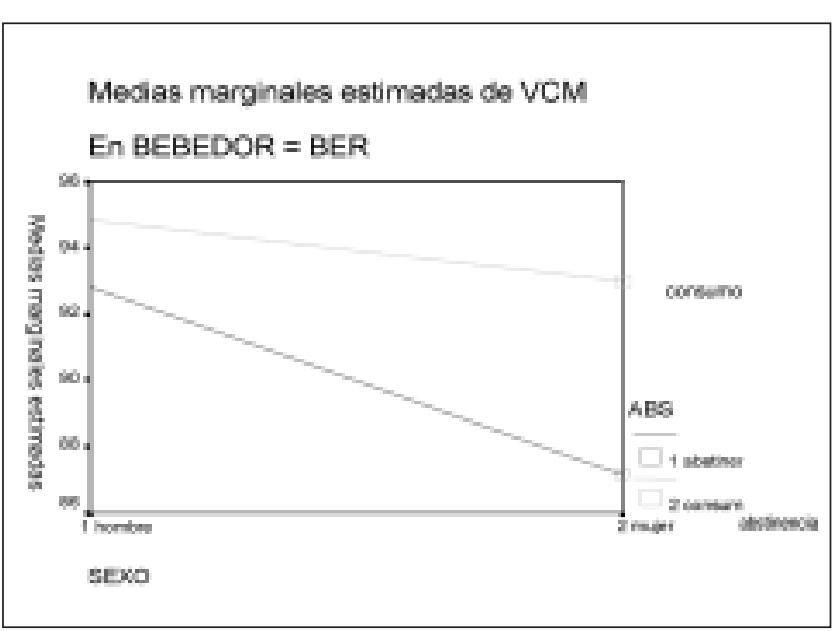

Gráfico II.

Medias marginales estimadas de VCM

En BEBEDOR $=\mathrm{B} \mid \mathrm{R}$

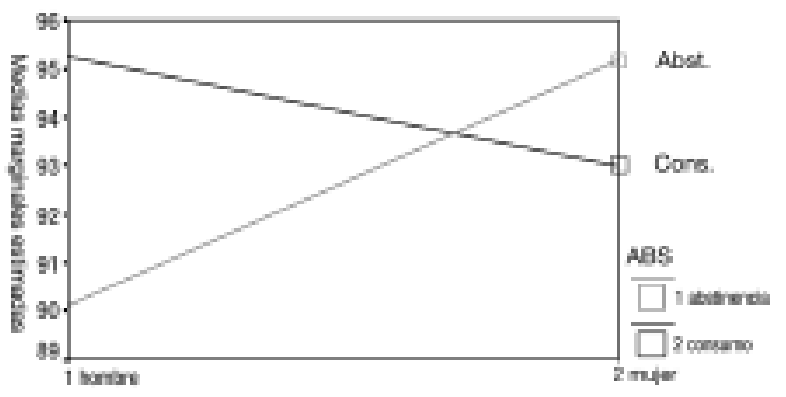

SEX0

BER: Bebedor Excesivo Regular.

BIR: Bebedor Intermitente.

\section{Gráfico III.}


Por una parte parece ser que entre las mujeres bebedoras regulares, es mayor la puntuación en VCM de aquellas que continúan consumiendo frente a las que permanecen abstinentes, no siendo tal diferencia significativa a nivel estadístico entre los varones, aunque se aprecia una tendencia en el mismo sentido que la de las mujeres.

Respecto a los consumidores intermitentes, los varones consumidores obtienen puntuaciones en VCM mayores estadísticamente que las obtenidas por los abstinentes, no siendo tal diferencia estadísticamente significativa entre las mujeres, llegando incluso a apreciarse una relación inversa.

Respecto al índice GGT, entre los varones no se establecen diferencias en las medidas obtenidas. Todos obtienen puntuaciones similares independientemente de si continúan o no consumiendo, de si han sido bebedores regulares o intermitentes e independientemente de ser mayores o no de 40 años. El mismo resultado se atribuye al grupo de mujeres.

Si no se establece la diferencia en función del sexo tampoco se encuentran diferencias significativas.

Parece ser que este índice pierde su valor conforme pasa el tiempo, ya que en estos momentos cualquiera de las variables manipuladas (edad, abstinencia, sexo y tipo de bebedor) no hacen variar las puntuaciones que se obtienen.

Nos resta comentar los valores de la variable CDT. En este caso separamos los resultados obtenidos antes del año 2000 de los obtenidos a lo largo de este año.

- CDT/edad*abs*sexo (es significativa la abstinencia)

- CDT/edad*abs*bebedor (es significativa la abstinencia)

- CDT/sexo*abs*bebedor (es significativa la abstinencia)

En este primer grupo los índices en CDT difieren significativamente entre los sujetos consumidores $y$ los ya abstinentes $\left(F_{1,62}=6,875 ; p=0,011 ; F_{1,62}=9,141\right.$; $\left.p=0,004 ; F_{1,62}=10,021 ; p=0,02\right)$, al igual que ocurría en el primer período temporal. Concretamente este índice continua siendo mayor entre los consumidores.

\section{- CDT (antes 2000) /Abst}

Abstinentes: Frecuencia 26 (valor medio 13'68 U/L). Consumidores: Frecuencia 45 (valor medio 27'86 $\mathrm{U} / \mathrm{L})$.
- CDT/sexo*abs (es significativa la abstinencia)

- CDT/abs*edad (es significativa la abstinencia)

-CDT/abs*bebedor (es significativa la abstinencia)

Lo mismo ocurre en el período posterior, también los sujetos consumidores obtienen mayores puntuaciones en CDT al compararlos con los sujetos abstinentes $\left(F_{1,16}=6,018 ; p=0,026 ; F_{1,15}=7,864 ; p=0,013\right.$; $\left.F_{1,14}=6,541 ; p=0,020\right)$.

\section{- CDT (después 2000) /Abst}

Abstinentes: Frecuencia 17 (valor medio 4'68 U/L). Consumidores: Frecuencia 3 (valor medio 7'63 .U/L)

Pero aunque pueden establecerse estas conclusiones, lo más adecuado sería conseguir un mayor número de mujeres en ambos casos, ya que en ocasiones los resultados pueden verse influidos por el menor número de éstas.

Asimismo otra recomendación de cara a mejorar y optimizar los resultados obtenidos sería el incrementar el número de sujetos bebedores intermitentes (BIR) pues al segmentar la población aparecen muchas categorías con un solo sujeto lo que invalida la variabilidad de la muestra, siendo uno de los factores a incidir en los problemas de homogeneidad.

\section{Análisis discriminante}

Otro de los objetivos del presente trabajo era determinar cual de los tres índices o la combinación de alguno de ellos era el más bueno para discriminar entre sujetos consumidores de alcohol de aquellos que ya no estaban consumiendo.

Por este motivo en el siguiente análisis discriminante se intentará determinar cual de los índices es el mejor en función de la variable ABSTINENCIA.

Se han realizado las pruebas primero considerando los tres índices, luego segmentando la muestra en función del sexo y finalmente en función del año de realización de la prueba.

Los resultados conseguidos para la primera determinación indican que el índice CDT es el más potente de los tres, pudiéndose utilizar sólo ya que el porcentaje de varianza que se explica de más al incluir VCM no es demasiado.

En este caso el papel de GGT es el menor de todos. Podemos concluir que es la CDT la variable que explica mayor cantidad de varianza entre los sujetos que se mantienen abstinentes y los que continúan consumiendo. Tabla III. 
Tabla III: Análisis discriminante antes del 2000.

\begin{tabular}{|l|c|c|c|c|}
\hline INDICES & $\begin{array}{c}\text { Correlación } \\
\text { canónica }\end{array}$ & $\begin{array}{c}\text { Lambda } \\
\text { de Wilks }\end{array}$ & $\begin{array}{c}\text { Coeficientes } \\
\text { estandarizados }\end{array}$ & centroides \\
\hline CDT & $52,9 \%$ & 0,720 & 0,957 & $-0,879$ \\
GGT & & $(p=0,000)$ & 0,186 & 0,439 \\
VCM & & 0,125 & \\
\hline CDT & $52,3 \%$ & 0,727 & 0,959 & $-0,865$ \\
VCM & & $(p=0,000)$ & 0,179 & 0,432 \\
\hline GGT & $20 \%$ & 0,960 & 0,357 & $-0,288$ \\
VCM & & $(p=0,000)$ & 0,845 & 0,144 \\
\hline CDT & $52,6 \%$ & 0,723 & 0,982 & $-0,873$ \\
GGT & & $(p=0,000)$ & 0,221 & 0,436 \\
\hline CDT & $51,7 \%$ & 0,733 & & $-0,851$ \\
& & & & 0,426 \\
\hline
\end{tabular}

Las tres juntas explican el $53 \%$ de la varianza, mientras que CDT sola explica el $52 \%$. AL comparar los resultados de los análisis discriminantes podemos concluir que la variable CDT sería suficiente para explicar más de la mitad de las diferencias entre los sujetos, no aportando prácticamente nada el resto de los índices evaluados. Cabe resaltar que la menor varianza viene explicada por el binomio GGT*VCM el cual únicamente permite detectar correctamente un 20\% de los sujetos.

El mismo resultado obtenemos al diferenciar por sexos, en ambos casos es la variable CDT la mejor predictora no resultando significativa la aportación del resto de índices. En este caso cabe resaltar que mientras que entre los varones la CDT explica un $50 \%$ de la varianza, entre las mujeres ella sola alcanza el $59 \%$.

Al diferenciar por año de realización de la prueba también se obtiene el mismo resultado, siendo asimismo la combinación $\mathrm{VCM}{ }^{*} \mathrm{GGT}$ la que peores resultados ofrece. Además el índice predice más (62,1\%) entre los sujetos del 2000 parece ser que el nuevo reactivo utilizado desde principios del 2000 es más sensible que el anterior

Al realizar los análisis discriminantes para la muestra 2 se aprecia el mismo resultado, continua siendo el índice CDT el mejor detector de los sujetos que consumen frente a los que no consumen.

En este caso CDT contribuye a explicar el $50 \%$ de la varianza explicada frente al $30 \%$ que explican conjuntamente VCM y GGT y frente al 55\% que explican los tres índices conjuntamente. Tabla IV

Al igual que en los casos anteriores al diferenciar o segmentar la muestra en función del año de recogida de la información (antes del 2000/a lo largo del 2000) se aprecia una mejora en cuanto al porcentaje de varianza que llega a explicar CDT, pasando de un $41 \%$ antes del 2000 a un $54 \%$ después de este. Si bien todos estos datos hay que tomarlos con precaución ya que sería conveniente incrementar el número de muestra de este segundo período.

Incluso para posteriores estudios, se podría completar el análisis estadístico con las curvas de ROC (Receiver Operating Characteristics) o incluso con un análisis bayesiano de sensibilidad y especificidad.

Tabla IV: Análisis discriminante después del 2000.

\begin{tabular}{|l|c|c|c|c|}
\hline INDICES & $\begin{array}{c}\text { Correlación } \\
\text { canónica }\end{array}$ & $\begin{array}{c}\text { Lambda } \\
\text { de Wilks }\end{array}$ & $\begin{array}{c}\text { Coeficientes } \\
\text { estandarizados }\end{array}$ & centroides \\
\hline CDT & $55,3 \%$ & 0,694 & 0,879 & $-0,067$ \\
GGT & & & 0,067 & 0,622 \\
VCM & & 0,508 & \\
\hline CDT & $55,3 \%$ & 0,695 & 0,882 & $-0,693$ \\
VCM & & & 0,495 & 0,621 \\
\hline GGT & $30,2 \%$ & 0,909 & $-0,180$ & $-0,331$ \\
VCM & & & 1,023 & 0,297 \\
\hline CDT & $50 \%$ & 0,750 & 1 & $-0,603$ \\
GGT & & & 0,049 & 0,540 \\
\hline CDT & $49,9 \%$ & 0,751 & & $-0,602$ \\
& & & & 0,539 \\
\hline VCM & $29,8 \%$ & 0,911 & & $-0,326$ \\
& & & & 0,292 \\
\hline
\end{tabular}

\section{CONCLUSIONES:}

La determinación sérica de la transferrina deficiente en carbohidratos (CDT), nos servirá para detectar consumos de etanol superiores a $50 \mathrm{gr} /$ día durante al menos una semana. Al normalizarse a los 15 días de la abstinencia, cualquier incremento posterior será indicativo de una recaída en el consumo, atendiendo a las mismas cantidades, por lo que no nos servirá para consumos esporádicos o inferiores a $50 \mathrm{gr}$.

A diferencia de la GGT o el VCM, no suele alterarse por otras enfermedades o por el consumo de fármacos $\mathrm{u}$ otras sustancias.

Aunque diversos estudios no han llegado a ponerse de acuerdo en la sensibilidad de la prueba, si que podemos afirmar que la CDT es la prueba de laboratorio más específica para determinar consumos de riesgo y para valorar la evolución del alcohólico abstinente en tratamiento.

Hemos realizado un análisis estadístico a partir de las determinaciones de laboratorio de VCM, GGT y CDT, para antes el año 2000 y para después del 2000 (año en que cambiamos de reactivo para CDT, siendo 
este último más sensible) y hemos encontrado como el VCM nos indica que existen diferencias significativas entre abstinentes y no abstinentes, independientemente del sexo o la edad. Aquí la aplicabilidad se complica cuando tomamos en cuenta si el bebedor es un bebedor intermitente o excesivo regular, apareciendo correlaciones inversas entre hombres y mujeres sobre todo entre los bebedores intermitentes.

En la GGT si que aparecen diferencias entre consumidores y abstinentes si no tenemos en consideración el sexo, en cambio no permite diferenciar en función de la edad ni de estar o no abstinentes cuando hacemos un análisis diferencial por sexos, lo cual representa un problema para su utilización como marcador del alcoholismo dependiendo del género.

A los 6 meses la GGT no obtiene diferencias significativas por lo que ya no es un buen indicador para el consumo, la GGT puede no alterarse con el consumo de etanol por lo que no es el mejor marcador para descubrir o controlar la dependencia alcohólica.

La CDT si que se muestra significativa para la abstinencia en todos los casos, sexo, tipo de bebedor, edad, por lo que estadísticamente los consumidores muestran niveles elevados de CDT para todas las muestras incluso las tomadas antes y después del 2000, tanto al inicio como a los 6 meses de tratamiento.

En el análisis discriminante podemos determinar además que la CDT, es el más potente de los 3 marcadores, ya que por el mismo explica un $51^{\prime} 7 \%$ de los casos frente al 52 ' $9 \%$ de (CDT + GGT + VCM). También después del 2000 el CDT contribuye a explicar el $50 \%$ de los casos frente al 30\% que explican conjuntamente VCM y GGT y frente al 55\% que explican los tres índices conjuntamente.

Por lo que podemos concluir que la CDT es la prueba que mayor peso estadístico tiene si pretendemos demostrar el consumo excesivo de alcohol, así como el reinicio en el consumo abusivo, a pesar de sus limitaciones, aunque sigue siendo de gran utilidad la GGT como marcador del alcoholismo.

\section{BIBLIOGRAFÍA:}

(1) Pagana. Guía de pruebas diagnósticas y de laboratorio. Mosby - Doyma libros S.A. Madrid 1996. Pags. 24-25; 125-127; 448-449; 563-566.

(2) Santes,J.; Pascual, F. Fenollar, J. Et alti. Utilidad de la transferrina deficiente en carbohidratos como marcador del consumo de alcohol frente a la gamma-glutamil-transferasa. Análisis. Clínicos 2000; 25 (2): 33-39.

(3) Rubio Valladolid, G. Abordaje del alcoholismo en atención primaria- El médico. 16-1-1998. P.p. 30-45.
(4) Saiz, P.A. et alti. Marcadores biológicos de la conducta alcohólica. Psiquiatría biológica. Vol.5 supl. 1 Doyma. Septiembre 1998 . P.p.37.

(5) Salesporo, M. (University of Helsinky - Finlandia) CDT and other marker of alcoholism. Libro de ponencias el XIX Congreso ISBRAS (Society for Biomedical Researsch on Alcoholism) June 27- July 2, 1998. Copenhague, Denmark.

(6) Gordon, H.M. Londres. CDT for monitoring drinking behaviour. Libro de ponencias el XIX Congreso ISBRAS (Society for Biomedical Researsch on Alcoholism) June 27- July 2, 1998. Copenhague, Denmark.

(7) Van Pelt, J. (Holanda) Is CDT wit the total transferrin concentration? Libro de ponencias el XIX Congreso ISBRAS (Society for Biomedical Researsch on Alcoholism) June 27- July 2 , 1998. Copenhague, Denmark.

(8) Raja K.O. et alti ( Real colegio de medicina y odontología de Londres). Serum transferrin receptor status in alcoholism. Libro de ponencias el XIX Congreso ISBRAS (Society for Biomedical Researsch on Alcoholism) June 27- July 2, 1998. Copenhague, Denmark.

(9) Sarkola, T. Et alti. Mean cell volume and gamma-glutamyl transferase are superior to carbohydrate-deficient transferrin and hemoglobin-acetaldehyde adducts in the follow-up of pregnant women with alcohol abuse. Acta Obstet Gynecol Scand, 2000 May, 79:5, 359-66.

(10) Salaspuro, M. Carbohydrate-deficient transferrin as compared to other markers of alcoholism: a systematic review. Alcohol. 1999. Nov, 19:3, 261-71.

(11) Chrostek L.; Szmitkowski, M. CDT (desialylated transferrin) a neww biochemical marker of alcohol abuse. Psychiatr Pol. 1999 Mar, 33:2, 189-201.

(12) BrÁthen, G. Et alti. Validity of carbohydrate deficient transferrin and other markers as diagnostic aids in the detection of alcohol related seizures. J. Neurol Neurosurg Psychiatry. 2000 Mar, 68:3, 342-8.

(13) Mundle, G. Et alti. Influence of age, alcohol consumption and abstinence on the sensitivity of carbohydratedeficient transferrin, gamma-glutamyltransferase and mean corpuscular volume. Alcohol, 1999. Sep, 34:5, 760-6.

(14) Meerkerk, GJ. Et alti. Comparing the diagnostic accuracy of carbohydrate-deficient transferrin, gamma-glutamyltransferrase, and mean cell volume in a general practice population. Alcohol Clin Exp Res, 1999 Jun, 23:6, 1052-9.

(15) Mikkelsen IM. Et alti. Carbohydrate-deficient transferrin: marker of actual alcohol consumption or chronic alcohol misuse?. Alcohol 1998 Nov, 33:6, 646-50.

(16) Yoshikawa, K. Y cols. Determination of carbohydratedeficient separated by lectin affinity chromatography for detecting chronic alcohol abuse. FEBS Lett, 1999 Sep, 458:2, 112-6.

(17) Varios autores. Tratado de Epidemiología clínica. DuPont Pharma, S.A. 1995. Pgas, 202- 203. 
